\title{
Classical simulation of fermionic linear optics augmented with noisy ancillas
}

\author{
Michał Oszmaniec* * Jan Gutt, and Marek Kuś \\ Center for Theoretical Physics, Polish Academy of Sciences, Al. Lotników 32/46, 02-668 Warszawa
}

\begin{abstract}
Fermionic linear optics is a model of quantum computation which is efficiently simulable on a classical probabilistic computer. We study the problem of a classical simulation of fermionic linear optics augmented with noisy auxiliary states. If the auxiliary state can be expressed as a convex combination of pure Fermionic Gaussian states, the corresponding computation scheme is classically simulable. We present an analytic characterisation of the set of convex-Gaussian states in the first non-trivial case, in which the Hilbert space of the ancilla is a four-mode Fock space. We use our result to solve an open problem recently posed by De Melo et al. [1] and to study in detail the geometrical properties of the set of convex-Gaussian states.
\end{abstract}

PACS numbers: 03.67.Hk, 03.67.Mn, 02.20.Tw

For any model of quantum computation it is vital to characterise its computational power. Probably the most important practical question is how a given model compares to universal classical, respectively quantum, computation. If protocols allowed by the model are efficiently simulable on a classical computer, the corresponding physical system may be accessible to numerical studies, but is unlikely to be a suitable candidate for a quantum computer. On the other hand, simulability by quantum circuits ensures that the underlying physics can be effectively studied using a quantum computer [2]. Lastly, if the resources provided by the model enable one to implement a universal quantum computation, the corresponding physical system is a candidate for the realization of a quantum computer.

Many physically motivated models of quantum computation are defined by specifying the available set of initial states, gates and measurements [3, 4]. The Fermionic Linear Optics (FLO) model of quantum computation, introduced in 5], is the fermionic analogue of well-known bosonic linear optics [6, 7]. In the FLO model the class of allowed operations includes: preparation of the vacuum state, free unitary fermionic evolution, and occupation number measurements. Fermionic linear optics describes systems of non-interacting fermions, i.e. fermionic systems that can be described exactly by the Bogolyubov mean field theory. The model of computation based on FLO alone is not computationally universal and can be effectively simulated by a classical probabilistic computer [5, 8]. Nevertheless, the physics beyond FLO is rich and captures a number of systems of interest for condensed matter physics, including Kitaev's Majorana chain [9], honeycomb model [10], $\nu=5 / 2$ fractional Quantum Hall systems [11]. These systems possess a topological order and can be used as fault-tolerant quantum memories [12] or for the Topological Quantum Computation (TQC) with Ising anyons [10]. This motivates an interest in extending FLO in such a way that the resulting model will become computationally universal. In the present paper

\footnotetext{
* oszmaniec@cft.edu.pl
}

we study the computational power of the model in which FLO is augmented with a noisy ancilla state. In this model, introduced in [1], the traditional scheme of FLO is extended by introducing additional fermionic modes in which, at the beginning of computation, one stores certain number of copies of a, perhaps noisy, ancilla state. Initial state of the system in the original "computational" modes remains the vacuum state and the class of allowed operations remains intact. This model of computation is analogous to other ancilla-assisted models of quantum computation (such as ancilla-assisted computation with Clifford gates 13, 14 or ancilla-assisted topological quantum computing with Ising anyons [15]). Depending on the properties of the auxiliary state it may be possible to implement, with the help of traditional FLO operations, gates that are necessary for computational universality on registers describing the actual computation. In [1] the authors showed that whenever the state of the ancilla is convex-Gaussian, i.e. can be written as a convex combination of projectors onto pure Gaussian states (see definition bellow), the corresponding model of computation remains classically simulable. On the other hand, some ancilla states [13] do promote the model to be computationally universal.

In the current paper we present a complete analytical characterisation of the set of convex-Gaussian states for a special case when the Fock space of the ancilla has four modes. This is the lowest-dimensional non-trivial case, as for two and three modes all even pure states are Gaussian [1]. Moreover, the four mode Fock space is of special relevance as some states from this space can be used to make FLO and TQC with Ising anyons computationally universal [15]. A particular example is a non-Gaussian pure state $\left|a_{8}\right\rangle$ (see definition below). We use our analytical criterion to show that for a noise strength $p \geq p_{c r}=\frac{8}{11}$, the depolarised state $\left|a_{8}\right\rangle$ becomes convex-Gaussian and thus useless for computational purposes. We thus settle the open problem posed in a recent paper by de Melo et. al. [1]. In addition to the exact analytical criterion we explore, with the use of group-theoretical methods and tools of entanglement theory, the geometry of the set of convex-Gaussian states in the space of all density operators in a four mode Fock space. 
We first introduce the necessary notation and briefly describe the FLO model of computation and its classical simulation. The Hilbert space describing a fermionic system whose particles can be in $d$ modes is a Fock space which we denote by Fock $\left(\mathbb{C}^{d}\right)$. On this space we have the action of the standard creation and annihilation operators: $a_{i}^{\dagger}, a_{i}, i=1, \ldots, d$, which satisfy canonical anticommutation relations. The whole Fock space is spanned by the set of orthonormal Fock states:

$$
\left|n_{1}, \ldots, n_{d}\right\rangle=\left(a_{1}^{\dagger}\right)^{n_{1}} \cdots\left(a_{d}^{\dagger}\right)^{n_{d}}|0\rangle,
$$

where $|0\rangle$ is the Fock vacuum. It what follows we will consider only even operators, i.e., operators commuting with the total parity operator $Q=\prod_{k=1}^{d}\left(\mathbb{I}-2 a_{k}^{\dagger} a_{k}\right)$. The operator $Q$ has eigenvalues \pm 1 . The Fock space decomposes onto eigenspaces of $Q$, Fock $\left(\mathbb{C}^{d}\right)=$ Fock $_{+}\left(\mathbb{C}^{d}\right) \oplus$ Fock $-\left(\mathbb{C}^{d}\right)$, where Fock $\left(\mathbb{C}^{d}\right)$ and Fock $-\left(\mathbb{C}^{d}\right)$ are spanned by Fock states with respectively even and odd number of excitations. The corresponding orthogonal projectors onto these subspaces are $\mathbb{P}_{ \pm}=\frac{1}{2}(\mathbb{I} \pm Q)$. It is convenient to introduce Majorana fermion operators $[1,16]: c_{2 k-1}=a_{k}+a_{k}^{\dagger}, c_{2 k}=i\left(a_{k}-a_{k}^{\dagger}\right), k=1, \ldots, d$. One checks that they are Hermitian and satisfy anticommutation relations $\left\{c_{k}, c_{l}\right\}=2 \delta_{k l}$. The operator $Q$ takes the from $Q=i^{d} \prod_{k=1}^{2 d} c_{k}$. An Hermitian operator $X$ is even if and only if it can be written as a polynomial in Majorana operators involving only monomials of even degree,

$$
X=\alpha_{0} \mathbb{I}+\sum_{k=1}^{d} i^{k} \sum_{1 \leq l_{1} \leq l_{2} \leq \ldots \leq l_{2 k} \leq 2 d} \alpha_{l_{1} l_{2} \ldots l_{2 k}} c_{l_{1}} c_{l_{2}} \ldots c_{l_{2 k}},
$$

where all the coefficients $\alpha_{0}$ and $\alpha_{l_{1} l_{2} \ldots l_{2 k}}$ are real. For an even mixed state $\rho$ the correlation matrix $M$ is defined by $M_{i j}=\frac{i}{2} \operatorname{Tr}\left(\rho\left[c_{k}, c_{l}\right]\right), k, l=1, \ldots, 2 d$; it is real and antisymmetric. Pure fermionic Gaussian states, which we denote by $\mathcal{G}$, are by definition [1], states for which the correlation matrix is orthogonal,

$$
\mathcal{G}=\left\{|\psi\rangle \in \mathcal{H}_{\text {Fock }}\left(\mathbb{C}^{N}\right) \mid M M^{T}=\mathbb{I}_{2 N}\right\} .
$$

Pure fermionic Gaussian states, by virtue of the fermionic Wick theorem [16], are fully determined by their correlation matrix $M$. A given mixed state $\rho$ is called convexGaussian if and only if it can be expressed as a convex combination of pure Gaussian states, $\rho=\sum_{i} p_{i}\left|\psi_{i}\right\rangle\left\langle\psi_{i}\right|$, where $\sum_{i} p_{i}=1$, and for each $\left|\psi_{i}\right\rangle \in \mathcal{G}$ is a pure Gaussian state. We denote the set of convex-Gaussian [17] states by $\mathcal{G}^{c}$ expressing the fact that this set is the convex hull of $\mathcal{G}$ in the space of Hermitian operators on Fock $\left(\mathbb{C}^{N}\right)$. Note that we have an analogy with the separability problem, where the class of separable mixed states is defined as the convex hull of the set of pure product states.

We now briefly recall the computation model based on fermionic linear optics introduced in [1]. The allowed operations in the model are: (i) preparation of the Fock vacuum $|0\rangle$, (ii) measurement of the occupation numbers $a_{k}^{\dagger} a_{k}=\frac{1}{2}\left(\mathbb{I}+i c_{2 k-1} c_{2 k}\right)$ for any mode $k$, (iii) evolution under the von Neumann equation, $\frac{d}{d t} \rho=-i[H, \rho]$, for time $t$. The Hamiltonian $H=i \sum_{k, l=1}^{2 N} h_{k l} c_{k} c_{l}$ is an arbitrary Hamiltonian quadratic in Majorana operators. Operations (i), (ii) and (iii) can be preformed in arbitrary order and may depend upon measurement results obtained during previous stages of the computation. The protocol concludes with the final measurement whose (binary) outcome is the result of the computation. The above model of computation can be efficiently simulated in polynomial time on a probabilistic classical computer (a classical computer having access to random bits). The proof relies on the fact that the state remains Gaussian along the computation and that there exist update rules for the correlation matrix $M$ that have low computational complexity. We now extend the above model by allowing (iv) multiple usage of the ancilla state $\rho$ that is stored in auxiliary $m$ modes ( $\rho$ is a state on Fock $\left(\mathbb{C}^{m}\right)$ ). If $k$ auxiliary states are available, the total Hilbert space of the system becomes Fock $\left(\mathbb{C}^{d}\right) \otimes\left(\text { Fock }\left(\mathbb{C}^{m}\right)\right)^{\otimes k}=\operatorname{Fock}\left(\mathbb{C}^{d+k \cdot m}\right)$ and we allow arbitrary operations of the form (ii) and (iii) to be performed on the initial state of the total system of the form $|0\rangle\langle 0| \otimes \rho^{\otimes k}$. The computation model (i-iv) can be effectively classically simulated if the auxiliary state $\rho$ is convex-Gaussian i.e. $\rho=\sum_{i} p_{i}\left|\psi_{i}\right\rangle\left\langle\psi_{i}\right|$ for $\left|\psi_{i}\right\rangle \in \mathcal{G}[1]$. This is the reason why the characterisation of the convexGaussian states $\mathcal{G}^{c}$ is important for this model of computation. The simulation scheme consists of sampling pure Gaussian states $\left\{\left|\psi_{i}\right\rangle\right\}$ according to the probability distribution $\left\{p_{i}\right\}$ followed by the classical simulation of the of the evolution of Gaussian states described in [5, 16]. We would like to point out that convex-Gaussian ancilla states lead to an effectively classically simulable model also when one replaces FLO with its dissipative counterpart, recently introduced in [8]. For this reason results presented in this work are also valid for the dissipative FLO.

We can now give a complete analytical characterisation of the set of convex-Gaussian states for the special case of Fock $\left(\mathbb{C}^{4}\right)$. Let $\rho$ be an arbitrary even mixed state on Fock $\left(\mathbb{C}^{4}\right)$ having the decomposition (2). Let $\rho_{+}=\mathbb{P}_{+} \rho \mathbb{P}_{+}, \rho_{-}=\mathbb{P}_{-} \rho \mathbb{P}_{-}$denote restrictions of $\rho$ to Fock $_{+}\left(\mathbb{C}^{4}\right)$ and Fock $-\left(\mathbb{C}^{4}\right)$, respectively. By $\tilde{X}$ we denote the "complex conjugate" of the operator $X$, i.e., an operator constructed from $X$ by changing all $i$ to $-i$ in the decomposition (2). Let us introduce nonnegative functions $C_{+}$and $C_{-}$that are the analogues of Ulhmann-Wooters concurrence describing entanglement in two qubit systems [18]. They are defined by

$$
C_{ \pm}(\rho)=\max \left\{0, \lambda_{1}^{ \pm}-\sum_{k=2}^{8} \lambda_{k}^{ \pm}\right\}
$$

where $\left(\lambda_{1}^{ \pm}, \lambda_{2}^{ \pm}, \ldots, \lambda_{8}^{ \pm}\right)$denote non-increasingly ordered eigenvalues of the operator $\sqrt{\rho_{ \pm} \tilde{\rho}_{ \pm}}$. Convex-Gaussianity 
of $\rho$ is characterised by the values of these generalised concurrences,

$$
\rho \text { is convex-Gaussian } \Longleftrightarrow C_{+}(\rho)=C_{-}(\rho)=0 .
$$

Application of methods from entanglement theory enable us to give a detailed description of the geometry of convex-Gaussian states in Fock $\left(\mathbb{C}^{4}\right)$ [19]. For a given state $\rho$, supported in Fock $\left(\mathbb{C}^{4}\right)$, we provide a measure of its distance to the set of convex-Gaussian states $\mathcal{G}^{c}$ in terms of Uhlmann fidelity with respect to the set $\mathcal{G}^{c}$. The following formula holds

$$
F_{\text {Gauss }}(\rho)=\max _{\sigma \in \mathcal{G}^{c}} F(\rho, \sigma)=\frac{1}{2}+\frac{1}{2} \sqrt{1-C_{+}^{2}(\rho)},
$$

where $F(\rho, \sigma)=(\operatorname{tr}[\sqrt{\sqrt{\rho} \sigma \sqrt{\rho}}])^{2}$ denotes Uhlmann fidelity between states $\rho$ and $\sigma$. Using Fuchs-van de Graaf inequalities [20] we bound the statistical (trace) distance [21] of any state $\rho$ on Fock $_{+}\left(\mathbb{C}^{4}\right)$ to the set of convexGaussian states, $D\left(\rho, \mathcal{G}^{c}\right)=\min _{\sigma \in \mathcal{G}^{c}} \frac{1}{2} \sqrt{\operatorname{tr}\left((\rho-\sigma)^{2}\right)}$, by

$$
1-\sqrt{F_{\text {Gauss }}(\rho)} \leq D\left(\rho, \mathcal{G}^{\text {conv }}\right) \leq \sqrt{1-F_{\text {Gauss }}(\rho)} .
$$

Inequalities (7) together with (6) show that for a non convex-Gaussian state $\rho$ supported in Fock $\left(\mathbb{C}^{4}\right)$ the generalised concurrence $C_{+}(\rho)$ can be used to assess the resilience of the property of being non convex-Gaussian against noise.

Before proceeding to the proofs of (5) and (6), we use (5) to give the noise threshold $p_{c r}$ above which a depolarisation of the state $\left|a_{8}\right\rangle \in$ Fock $\left(\mathbb{C}^{4}\right)$ becomes convexGaussian. In other words we consider a state

$$
\rho(p)=(1-p)\left|a_{8}\right\rangle\left\langle a_{8}\right|+p \frac{\mathbb{I}}{16},
$$

where $p \in[0,1], \mathbb{I}$ is the identity operator, and

$$
\left|a_{8}\right\rangle\left\langle a_{8}\right|=\frac{1}{16}\left(\mathbb{I}+S_{1}\right)\left(\mathbb{I}+S_{2}\right)\left(\mathbb{I}+S_{3}\right)(\mathbb{I}+Q)
$$

is a pure state which can be used to implement a CNOT gate that is needed to promote FLO to be computationally universal [1, 15]. The problem of finding $p_{c r}$ was considered in [5] where authors showed that $\rho(p)$ is non convex-Gaussian for $p \leq \frac{8}{15}$ and is convex-Gaussian for $p \geq \frac{8}{9}$. Application of (5) to (8) is straightforward because $\left|a_{8}\right\rangle \in \operatorname{Fock}\left(\mathbb{C}^{4}\right)$ and $\tilde{\rho}(p)=\rho(p)$. Simple algebra shows that $\rho(p)$ is convex-Gaussian if and only if $p \geq \frac{8}{11}=p_{c r}$. This result is particularly interesting as it opens a possibility for existence of more noise-resilient protocols of distillation of the state pure $\left|a_{8}\right\rangle$ from copies of a noisy state $\rho(p)$ via FLO or TQC with Ising Anyons (the protocol based on TQC introduced in [15] works for $p \leq 0.4)$.

We now prove our criterion (5D). Let us first note that pure fermionic Gaussian states have a fixed parity. In other words: $\mathcal{G}=\mathcal{G}_{+} \cup \mathcal{G}_{-}$, where $\mathcal{G}_{ \pm} \subset \operatorname{Fock}_{ \pm}\left(\mathbb{C}^{m}\right)$. For this reason it is enough to consider the problem of convex-Gaussianity separately on Fock $\left(\mathbb{C}^{m}\right)$. In other words an even state $\rho$ is convex-Gaussian if and only if both $\rho_{+}$and $\rho_{-}$are convex-Gaussian. We show below that there exist antiunitary operators $\theta_{ \pm}$, each acting on Fock $_{ \pm}\left(\mathbb{C}^{4}\right)$, such that

$$
|\psi\rangle \in \mathcal{G}_{ \pm} \Longleftrightarrow C_{ \pm}(|\psi\rangle)=\left|\left\langle\psi \mid \theta_{ \pm} \psi\right\rangle\right|=0 .
$$

We can now use the Uhlmann-Wooters construction [18] to compute the convex roof extension of $C_{ \pm}, C_{ \pm}(\sigma)=$ $\inf _{\sum\left|\psi_{i}\right\rangle\left\langle\psi_{i}\right|=\sigma}\left(\sum_{i} C_{ \pm}\left(\left|\psi_{i}\right\rangle\right)\right)$, for $\sigma$ a non-negative operator on Fock ${ }_{ \pm}\left(\mathbb{C}^{m}\right)$. From the definition of the convex roof extension and the discussion above we have that $\rho$ is convex-Gaussian if and only if $C_{+}\left(\rho_{+}\right)=C_{-}\left(\rho_{-}\right)=0$. Explicit formulas for $C_{ \pm}\left(\rho_{ \pm}\right)$are given by (44), where $\left(\lambda_{1}^{ \pm}, \lambda_{2}^{ \pm}, \ldots, \lambda_{8}^{ \pm}\right)$denote non-increasingly ordered eigenvalues of the operator $\sqrt{\rho_{ \pm} \theta_{ \pm} \rho_{ \pm} \theta_{ \pm}}$[18]. From the details of the Uhlmann-Wooters construction it follows that for convex-Gaussian states supported in Fock ${ }_{ \pm}\left(\mathbb{C}^{4}\right)$ we need at most $\mathcal{N}=8$ pure Gaussian states in the convex decomposition (this is a consequence of the fact that Hadamard matrices exist in dimension 8 which is the dimension of $\mathrm{Fock}_{+}\left(\mathbb{C}^{4}\right)$ ). Consequently, for an arbitrary convex-Gaussian state in Fock $\left(\mathbb{C}^{4}\right)$ this number equals $\mathcal{N}=16$, much smaller than the upper bound $\tilde{\mathcal{N}}=48$ obtained in [1]. The existence of antiunitary operators $\theta_{ \pm}$follows from group-theoretical interpretation of pure Gaussian states $\mathcal{G}_{ \pm}$. The group of Bogolyubov transformations is precisely $\operatorname{Spin}(2 m)$, a compact semisimple Lie group. The Hilbert space Fock $\left(\mathbb{C}^{m}\right)$ decomposes into two irreducible representations of $\operatorname{Spin}(2 m)$ : Fock $_{+}\left(\mathbb{C}^{m}\right)$ and Fock $\left(\mathbb{C}^{m}\right)$ respectively. Sets of pure Gaussian states $\mathcal{G}_{ \pm}$are precisely the "highest weight" orbits of this group in Fock ${ }_{ \pm}\left(\mathbb{C}^{m}\right)$ 22]. Semi-simple compact Lie groups $K$ and irreducible representations $\mathcal{H}^{\lambda}$ 23] admitting an antiunitary operator $\theta: \mathcal{H}^{\lambda} \rightarrow \mathcal{H}^{\lambda}$ detecting the orbit through the highest-weight vector had been classified in [24]. In order to guarantee the existence of such $\theta$ it suffices to check that the following decomposition holds:

$$
\mathcal{H}^{\lambda} \vee \mathcal{H}^{\lambda}=\mathcal{H}^{2 \lambda} \oplus \mathcal{H}_{0},
$$

where $\vee$ denotes the symmetric tensor product of Hilbert spaces (thus, $\mathcal{H}^{\lambda} \vee \mathcal{H}^{\lambda}$ becomes a representation of $K$ ), $\mathcal{H}^{2 \lambda}$ is an irreducible representation of $K$ characterised by the highest weight $2 \lambda$, and $\mathcal{H}_{0}$ is a trivial (one dimensional) representation of $K$. From the construction of an antiunitary $\theta$ presented in 24] it follows that it is $K$ invariant: $k \theta k^{\dagger}=\theta$, for all elements $k$ of the Lie group $K$. In our case we have $K=\operatorname{Spin}(8)$ and $\mathcal{H}^{\lambda}=$ Fock $_{+}\left(\mathbb{C}^{4}\right)$ or $\mathcal{H}^{\lambda}=$ Fock $_{-}\left(\mathbb{C}^{4}\right)$. For these particular representations the decomposition (10) indeed holds (see for example [22]) and thus existence of Spin (8)-invariant antiunitaries $\theta_{ \pm}$is guaranteed. We conclude the proof of (5) showing that $\theta_{ \pm} X \theta_{ \pm}=\tilde{X}[25]$ for every operator $X$ supported in either Fock $+\left(\mathbb{C}^{4}\right)$ or Fock $\left(\mathbb{C}^{4}\right)$. We present 
here a proof only for the even case. The desired property of $\theta_{+}$follows from its invariance under the action of Spin (8). Since Spin (8) is generated by anti-Hermitian operators $c_{i} c_{j}$, it follows that $c_{i} c_{j} \theta_{+}=\theta_{+} c_{i} c_{j}$ for every pair of Majorana operators. Using the fact that $\theta_{+}$satisfies (as every antiunitary operator) $\theta_{+}^{2}=\mathbb{I}, \theta i=-i \theta$ and noting that every operator $X$ with support in Fock $\left(\mathbb{C}^{4}\right)$ is an even operator (and thus has a decomposition (2)) proves $\theta_{ \pm} X \theta_{ \pm}=\tilde{X}$. This concludes the proof of (5).

We can now describe the action of $\theta_{+}$on pure states: expressing $c_{i} c_{j}$ in terms of creation and annihilation operators, and using antilinearity of $\theta_{+}$, one checks that $a_{k} a_{l} \theta_{+}=\theta_{+} a_{k}^{\dagger} a_{l}^{\dagger}$, together with its conjugate $a_{k}^{\dagger} a_{l}^{\dagger} \theta_{+}=$ $\theta_{+} a_{k} a_{l}$. Furthermore, we have $a_{k}^{\dagger} a_{l}^{\dagger} \theta_{+}|0\rangle=\theta_{+} a_{k} a_{l}|0\rangle=$ 0 so that $\theta_{+}|0\rangle$ is a maximally occupied state. We can fix the phase ambiguity in $\theta_{+}$to ensure $\theta_{+}|0\rangle=|1,1,1,1\rangle$. It then follows that $\theta_{+}$sends a state $\left|n_{1}, n_{2}, n_{3}, n_{4}\right\rangle$ in Fock $_{+}\left(\mathbb{C}^{4}\right)$ to $(-1)^{N / 2}\left|\bar{n}_{1}, \bar{n}_{2}, \bar{n}_{3}, \bar{n}_{4}\right\rangle$, where $\overline{0}=1, \overline{1}=0$ and $N=n_{1}+n_{2}+n_{3}+n_{4}$. An analogous formula holds for the action of $\theta_{-}$on Fock $-\left(\mathbb{C}^{4}\right)$, so that we can write universally $\theta_{ \pm}\left|n_{1}, n_{2}, n_{3}, n_{4}\right\rangle=(-1)^{\lfloor N / 2\rfloor}\left|\bar{n}_{1}, \bar{n}_{2}, \bar{n}_{3}, \bar{n}_{4}\right\rangle$, thus allowing one to view $\theta_{ \pm}$as implementing an excitationhole duality.

In order to verify (6) we describe the geometry of the action of $\operatorname{Spin}(8)$ on pure states in Fock $\left(\mathbb{C}^{4}\right)$. We show that the concurrence of a pure state, $C_{+}(|\psi\rangle)=$ $\left|\left\langle\psi \mid \theta_{+} \psi\right\rangle\right|$, parametrises orbits of Spin (8) in Fock $+\left(\mathbb{C}^{4}\right)$. It is known [26] that every state $|\psi\rangle \in \operatorname{Fock}_{+}\left(\mathbb{C}^{4}\right)$ can be written, up to phase, as a combination

$$
|\psi\rangle=\sqrt{1-a^{2}}\left|\psi_{1}\right\rangle+i a\left|\psi_{2}\right\rangle
$$

where $0 \leq a \leq \frac{1}{\sqrt{2}}$, and $\left|\psi_{1}\right\rangle,\left|\psi_{2}\right\rangle$ are orthogonal states satisfying $\theta_{+}\left|\psi_{\alpha}\right\rangle=\left|\psi_{\alpha}\right\rangle, \alpha=1,2$ (the latter condition corresponds to $\left|\psi_{\alpha}\right\rangle\left\langle\psi_{\alpha}\right|$ having real coefficients in the decomposition (10) ). Furthermore, by [24], property (10) implies that $\operatorname{Spin}(8)$ acts transitively on orthogonal pairs $\left|\psi_{1}\right\rangle,\left|\psi_{2}\right\rangle$ as above, whence we conclude that the set of all states $|\psi\rangle$ corresponding to a given value of $a$ is an orbit. In particular, $a=\frac{1}{\sqrt{2}}$ describes Gaussian states, while $a=0$ is the orbit of $\left|a_{8}\right\rangle$ [27]. Note that $C_{+}(|\psi\rangle)=$ $1-2 a^{2}$, so that $a$ is uniquely determined by $C_{+}(|\psi\rangle)$. It is also possible to re-express the state $|\psi\rangle$, up to a phase, as a combination of orthogonal Gaussian states (a generalised Schmidt decomposition). Namely, setting $\left|\psi_{G}\right\rangle=\frac{1}{\sqrt{2}}\left(\left|\psi_{1}\right\rangle-i\left|\psi_{2}\right\rangle\right)$, we have

$$
|\psi\rangle=\sqrt{1-p^{2}}\left|\psi_{G}\right\rangle+p \theta_{+}\left|\psi_{G}\right\rangle
$$

where $p=\frac{1}{\sqrt{2}}\left(\sqrt{1-a^{2}}-a\right)$. Gaussianity and orthogonality of $\left|\psi_{G}\right\rangle, \theta_{+}\left|\psi_{G}\right\rangle$ is immediately verified. It is important to remark that in (12) the phase of $\left|\psi_{G}\right\rangle$ does matter (unless $|\psi\rangle$ is itself Gaussian).

The proof of (6) relies on Theorem 2 of [28] which, for our purposes, states that $F_{\text {Gauss }}(\rho)$ can be described as a convex roof extension of a function defined on pure states:

$$
F_{\text {Gauss }}(\rho)=\sup _{\sum p_{i}}\left|\psi_{i}\right\rangle\left\langle\psi_{i}\right|=\rho\left(\sum_{i} p_{i} F_{\text {Gauss }}\left(\left|\psi_{i}\right\rangle\right)\right),
$$

where $F_{\text {Gauss }}(|\psi\rangle)=\max _{|\phi\rangle}\left\langle_{\phi \mid \in \mathcal{G}} F(|\psi\rangle\langle\psi|,| \phi\rangle\langle\phi|)\right.$. Using the fact that for $|\psi\rangle \in \operatorname{Fock}_{+}\left(\mathbb{C}^{4}\right)$ we have the decomposition (12) we find that $F_{\text {Gauss }}(|\psi\rangle)=\tilde{F}\left(C_{+}(|\psi\rangle)\right)$, where $\tilde{F}(x)=\frac{1}{2}+\frac{1}{2} \sqrt{1-x^{2}}$ is a strictly concave decreasing function on the interval $[0,1]$. Let $\rho=\sum_{i} p_{i}\left|\psi_{i}\right\rangle\left\langle\psi_{i}\right|$ be the optimal decomposition of $\rho$ leading to (4). From [18] it follows the all pure states in this decomposition have the same value of the generalised concurrence i.e. $C_{+}\left(\left|\psi_{i}\right\rangle\right)=C_{+}(\rho)$. Using this fact and (13) we have $F_{\text {Gauss }}(\rho) \geq \tilde{F}\left(C_{+}(\rho)\right)$. On the other hand, by concavity of $\tilde{F}$ we have

$\sum_{i} p_{i} F_{\text {Gauss }}\left(\left|\psi_{i}\right\rangle\right) \leq \tilde{F}\left(\sum_{i} p_{i} C_{+}\left(\left|\psi_{i}\right\rangle\right)\right)=\tilde{F}\left(C_{+}(\rho)\right)$,

which concludes the proof of (6).

Summarising, we have presented a complete analytical characterisation (5) of convex-Gaussian states in the four-mode fermionic Fock space Fock $+\left(\mathbb{C}^{4}\right)$. Using methods taken from entanglement theory and theory of Lie groups we described quantitatively (see (6) and (17) how the property of being non convex-Gaussian is resilient to noise. These results have immediate consequence for the computation power of models in which FLO or TQC with Ising anyons are assisted with a noisy ancilla state. This follows form the fact that computations augmented with convex-Gaussian states are classically simulable. We have used our methods to give a precise value of the noise threshold $p_{c r}=\frac{8}{11}$ above which the state $\left|a_{8}\right\rangle$ (used to make FLO or TQC computationally universal [1]), when depolarised, becomes convex-Gaussian. This result is especially interesting as the threshold value $p_{c r}$ is much higher than previously known lower bounds. This opens a possibility for the existence of $\left|a_{8}\right\rangle$-distillation protocols based on FLO or TQC that are much more noise tolerant than the currently known ones. It would be also intriguing to explore, using the analogy with entanglement theory, the possible resource theory based on FLO or TQC with Ising anyons. However, preliminary studies show that a "naive" application of the entanglement purification protocol [29] based on the generalised Schmidt decomposition (12) is not applicable. In the future we would also like to characterise the set of convex-Gaussian states defined on Fock $\left(\mathbb{C}^{m}\right)$, for $m>4$. To tackle this problem we plan to develop a theory of non-linear witnesses introduced in [30].

\section{ACKNOWLEDGMENTS}

We would like to thank Piotr Ćwikliński, Ravindra Witold Chhajlany and Remigiusz Augusiak for fruitful 
discussions. The support of the ERC grant QOLAPS is gratefully acknowledged. MO and MK acknowledge the support of Polish National Science Centre grant under the contract number DEC-2011/01/M/ST2/00379. MK acknowledges the support of COST Action MP 1006.
[1] F. de Melo, P. Ćwikliński, and B. M. Terhal, New Journal of Physics 15, 013015 (2013).

[2] R. P. Feynman, International journal of theoretical physics 212016C.(198ג)

[3] D. Gottesman, arXiv preprint quant-ph/9807006 (1998)

[4] H. J. Briegel and R. Raussendorf, Physical Review Letters 86 (2003).

[5] B. M. Terhal and D. P. DiVincenzo, Physical Review A 65, 032325 (2002)

[6] P. Kok, W. J. Munro, K. Nemoto, T. C. Ralph, J. P. Dowling, and G. Milburn, Reviews of Modern Physics 79, 135 (2007).

[7] S. Aaronson and A. Arkhipov, in (ACM, 2011) pp. 333-342. Bravyi and and R. arXiv preprint arXiv:1112.2184 (2011).

[9] A. Y. Kitaev, Physics-Uspekhi 44, 131 (2001)

[10] A. Kitaev, Annals of Physics 321, 2 (2006)

[11] J. Alicea, Reports on Progress in Physics 75, 076501 (2012)

[12] L. Mazza, M. Rizzi, M. D. Lukin, and J. I. Cirac, Physical Review B 88, 205142 (2013)

[13] S. Bravyi and A. Kitaev, Physical Review A 71, 022316 (2005)

[14] V. Veitch, S. H. Mousavian, D. Gottesman, and J. Emerson, New Journal of Physics 16, 013009 (2014)

[15] S. Bravyi, Physical Review A 73, 042313 (2006).

[16] S. Bravyi, arXiv preprint quant-ph/0404180 (2004).

[17] It is important to underline the difference between convex-Gaussian states and general Gaussian states. The latter class consists of states of the form $\rho=K$. $\exp \left(\sum_{i \neq j} h_{i j} c_{i} c_{j}\right)$, where $K$ is a normalisation constant. Every Gaussian state is convex-Gaussian, but the converse is not true.

[18] A. Uhlmann, arXiv preprint quant-ph/9909060 (1999).

[19] Analogous results can be easily derived for convexGaussian states in Fock- $\left(\mathbb{C}^{4}\right)$ and general even states $\rho$ in Fock $\left(\mathbb{C}^{4}\right)$, but we do not present them here for simplicity.

Information Theory, IEEE Transactions on 45, 1216 (1999)

[21] I. Bengtsson and K. Zyczkowski, Geometry of quantum states: an introduction to quantum entanglement (Cambridge University Press, 2006).

[22] L. Manivel, SIGMA 5, 078 (2009)

[23] Irreducible representations of semi-simple Lie groups $K$ are characterised by a so-called highest weight $\lambda$. The highest weight $\lambda$ is a generalisation of the "total spin" irreducible representations of $S U(2)$. For a comprehensive coimatidng tion to the representation theory of semi-simple Lie groups see [31].

[24] M. Oszmaniec and M. Kuś, Journal of Physics A: Mathematical and Theoretical 45, 244034 (20

[25] In fact one can combine antiunitary operators $\theta_{ \pm}$to get an antiunitary operator $\tilde{\theta}$ acting on the total Fock space and having similar properties. For simplicity we do not present this construction here.

[26] M. Kuś and I. Bengtsson, Phys. Rev. A 80, 022319 (2009).

[27] For intermediate values of $a$, the presentation in terms of $\left|\psi_{1}\right\rangle,\left|\psi_{2}\right\rangle$ is unique up to an overall sign; for $a=\frac{1}{\sqrt{2}}$ it is unique up to rotation in the plane spanned by $\left|\psi_{1}\right\rangle,\left|\psi_{2}\right\rangle$; for $a=0$ only $\left|\psi_{1}\right\rangle$ is relevant, and is unique up to sign. Accordingly the real dimension of the orbit is 7 for $a=0$, 12 for $a=\frac{1}{\sqrt{2}}$ and 13 for intermediate values of $a$.

[28] A. Streltsov, H. Kampermann, and D. Bruß, New Journal of Physics 12, 123004 (2010).

[29] C. H. Bennett, H. J. Bernstein, S. Popescu, and B. Schumacher, Physical Review A 53, 2046 (1996).

[30] M. Oszmaniec and M. Kuś, arXiv preprint arXiv:1312.7359 (2013),

[31] B. Hall, Lie groups, Lie algebras, and representations: an elementary introduction, Vol. 222 (Springer, 2003). 